\title{
DELAY-DEPENDENT ROBUST STABILIZATION OF UNCERTAIN DISCRETE-TIME STATE-DELAYED SYSTEMS
}

\author{
Young Sam Lee* Wook Hyun Kwon ${ }^{*, 1}$ \\ * Engineering Research Center for Advanced Control and \\ Instrumentation, \\ School of Electrical Engineering and Computer Science, \\ Seoul National Univ., Seoul, 151-742, Korea
}

\begin{abstract}
This paper concerns a problem of robust stabilization of a class of discrete-time systems with norm-bounded parameter uncertainty and unknown constant delay. A new delay-dependent stabilization condition using a memoryless controller is formulated in terms of matrix inequalities. An algorithm involving convex optimization is proposed to design a controller guaranteeing a suboptimal maximal delay such that the system can be stabilized for all admissible uncertainties. Numerical examples are given to illustrate the proposed results.
\end{abstract}

Keywords: Delay-dependent, Discrete system, Time-delay, Norm-bounded uncertainty

\section{INTRODUCTION}

During the last decades, considerable attention has been devoted to the problem of stability analysis and controller design for time-delay systems. Especially, in accordance with the advance of robust control theory, a number of robust stabilization methods have been proposed for uncertain time-delay systems.

The existing robust stabilization results for timedelay systems can be classified into two types: delayindependent stabilization (Phoojaruenchanachai and Furuta, 1992)-(Kim et al., 1996) and delay-dependent stabilization (Niculescu et al., 1994)-(Fu et al., 1998). The delay-independent stabilization provides a controller which can stabilize a system irrespective of the size of the delay. On the other hand, the delaydependent stabilization is concerned with the size of the delay and usually provides an upper bound of the delay such that the closed-loop system is stable for any delay less than the upper bound. While the delayindependent stabilization has been extensively studied by many researchers for the last decades, the study for

1 To whom all correspondence should be addressed: address : San 56-1 Shillim-dong, Kwanak-ku, Seoul 151-742, Korea., phone: +82-2-880-7307, fax: +82-2-871-7010, email: whkwon@cisl.snu.ac.kr the delay-dependent stabilization is relatively new and still under progress (Niculescu et al., 1998). In general, the delay-dependent stabilization is considered less conservative than the delay-independent case.

Since most physical systems evolve in continuoustime, it is natural that theories for stability analysis and controller synthesis are mainly developed for continuous-time. However, it is is more reasonable that one should use a discrete-time approach for that purpose because the controller is usually implemented digitally. Despite this significance mentioned, less attention has been paid to discrete-time systems with delays (Kapila and Haddad, 1998)-(Mahmoud, 2000). It is mainly due to the fact that the delay-difference equations with known delays can be converted into a higher-order delayless system by augmentation approach. However, for systems with large known delay amounts, this scheme will lead to large-dimensional systems. Furthermore, for systems with unknown delay the augmentation scheme is not applicable.

In this paper, a new delay-dependent robust stabilization condition using a memoryless controller is presented for uncertain discrete state-delayed systems. An algorithm involving convex optimization is proposed to design a controller guaranteeing a suboptimal 
maximal delay such that the system can be stabilized for all admissible uncertainties. Since the existing stabilization methods for discrete-time delay systems are mostly delay-independent ones, the results developed in this paper will be as much meaningful.

The organization of the paper is as follows. In Section 2 , the problem to be solved is formulated and preliminary results are given. In Section 3, nominal statedelayed systems without uncertainties are considered first and stability analysis and stabilization results are presented. Then, Section 4 deals with uncertain timedelay systems and the results of the previous section are extended to robust stability and stabilization conditions. In Section 5, numerical examples are given to illustrate the proposed results and finally Section 6 makes conclusions.

\section{PROBLEM STATEMENT AND PRELIMINARIES}

Consider the following uncertain discrete-time statedelayed systems

$$
\begin{aligned}
x(k+1)= & {[A+D \Delta(k) E] x(k) } \\
& +\left[A_{1}+D \Delta(k) E_{1}\right] x(k-h) \\
& +\left[B+D \Delta E_{b}\right] u(k) \\
x(k)= & \phi(k), \quad-h \leq k \leq 0
\end{aligned}
$$

where $x(k) \in R^{n}$ is the state, $u(k) \in R^{m}$ is the control, $h$ is a positive integer representing the amount of delay, $\phi(\cdot)$ is the initial condition, $A, A_{1}, B, D$, $D_{1}, E, E_{1}$, and $E_{b}$ are real constant matrices with appropriate dimensions. $\Delta(k) \in R^{\alpha \times \beta}$ is a timevarying uncertainty of the form

$$
\Delta(k)=\operatorname{diag}\left\{\Delta_{1}(k), \cdots, \Delta_{r}(k)\right\}
$$

where

$$
\Delta_{i}^{T}(k) \Delta_{i}(k) \leq I, \quad i=1, \cdots, r .
$$

We are interested in designing a memoryless statefeedback controller

$$
u(k)=K x(k)
$$

where $K \in R^{m \times n}$ is a constant gain matrix. Our aim is to develop a delay-dependent robust stabilization method which provides a controller gain $K$ as well as an upper bound $\bar{h}$ of the delay such that the closedloop system is stable for any $h$ satisfying $0 \leq h \leq \bar{h}$ and for all admissible uncertainties.

In obtaining the main results of this paper, the following upper bound for the inner product of two vectors plays an important role:

$$
-2 a^{T} \mathcal{N} b \leq \inf _{X, Y, Z}\left[\begin{array}{cc}
X & Y-\mathcal{N} \\
Y^{T}-\mathcal{N} & Z
\end{array}\right]
$$

where

$$
\left[\begin{array}{cc}
X & Y \\
Y^{T} & Z
\end{array}\right]
$$

and $I$ denotes an identity matrix with an appropriate dimension. Extending this idea, we have the following lemma.
Lemma 1. Assume that $a(\cdot) \in R^{n_{a}}, b(\cdot) \in R^{n_{b}}$, and $\mathcal{N}(\cdot) \in R^{n_{a} \times n_{b}}$ are defined on the interval $\Omega$. Then, for any matrices $X \in R^{n_{a} \times n_{a}}, Y \in R^{n_{a} \times n_{b}}$ and $Z \in R^{n_{b} \times n_{b}}$, the following holds:

$$
\begin{aligned}
& -2 \sum_{j} a^{T}(j) \mathcal{N} b(k) \\
& \leq \sum_{j}\left[\begin{array}{l}
a(j) \\
b(j)
\end{array}\right]^{T}\left[\begin{array}{cc}
X & Y-\mathcal{N} \\
Y^{T}-\mathcal{N}^{T} & Z
\end{array}\right]\left[\begin{array}{l}
a(j) \\
b(j)
\end{array}\right]
\end{aligned}
$$

where

$$
\left[\begin{array}{cc}
X & Y \\
Y^{T} & Z
\end{array}\right] \geq 0
$$

Lemma 2. Let $D, E$, and $\Delta$ be real matrices of appropriate dimensions with $\Delta=\operatorname{diag}\left\{\Delta_{1}, \cdots, \Delta_{r}\right\}$, $\Delta_{i}^{T} \Delta_{i} \leq I_{n_{i}}, i=1, \cdots, r$. Then, for any real matrix $\Lambda=\operatorname{diag}\left\{\lambda_{1} I, \cdots, \lambda_{r} I\right\}>0$, the following inequalities will be true.

$$
\begin{aligned}
& D \Delta E+E^{T} \Delta^{T} D^{T} \leq D \Lambda D^{T}+E^{T} \Lambda^{-1} E \\
& D \Delta E+E^{T} \Delta^{T} D^{T} \leq D \Lambda^{-1} D^{T}+E^{T} \Lambda E .
\end{aligned}
$$

Proof: From the fact $\left(\Lambda^{\frac{1}{2}} D^{T}-\Lambda^{-\frac{1}{2}} \Delta E\right)^{T}\left(\Lambda^{\frac{1}{2}} D^{T}-\right.$ $\left.\Lambda^{-\frac{1}{2}} \Delta E\right) \geq 0$, it follows that

$$
D \Delta E+E^{T} \Delta^{T} D^{T} \leq D \Lambda D^{T}+E^{T} \Delta^{T} \Lambda^{-1} \Delta E .
$$

For $\Delta$ and $\Lambda$, we have the relation

$$
\begin{aligned}
\Delta^{T} \Lambda^{-1} \Delta & =\operatorname{diag}\left\{\lambda_{1}^{-1} \Delta_{1}^{T} \Delta_{1}, \cdots, \lambda_{r}^{-1} \Delta_{r}^{T} \Delta_{r}\right\} \\
& \leq \operatorname{diag}\left\{\lambda_{1}^{-1} I_{n_{1}}, \cdots, \lambda_{r}^{-1} I_{n_{r}}\right\}=\Lambda^{-1} .
\end{aligned}
$$

Hence, $E^{T} \Delta^{T} \Lambda^{-1} \Delta E \leq E^{T} \Lambda^{-1} E$. From this, (8) follows. The proof of $(9)$ starts from the fact $\left(\Lambda^{-\frac{1}{2}} D^{T}-\right.$ $\left.\Lambda^{\frac{1}{2}} \Delta E\right)^{T}\left(\Lambda^{-\frac{1}{2}} D^{T}-\Lambda^{\frac{1}{2}} \Delta E\right) \geq 0$. The remaining procedure is quite clear, hence omitted. This completes the proof.

\section{STABILITY AND STABILIZATION FOR NOMINAL SYSTEMS}

Let us consider a nominal state-delayed system

$$
\begin{aligned}
x(k+1) & =A x(k)+A_{1} x(k-h)+B u(k), \\
x(k) & =\phi(k), \quad k \in[-h, 0] .
\end{aligned}
$$

We start with stability analysis of the unforced system (10) with $u(k)=0$. The following theorem presents a delay-dependent stability condition, which is the starting point of our further developments.

Theorem 1. If there exist $P, Q, X, Y$ and $Z$ such that

$$
\begin{gathered}
{\left[\begin{array}{cccc}
(1,1) & -Y & A^{T} P & (1,4) \\
-Y^{T} & -Q & A_{1}^{T} P & \bar{h} A_{1}^{T} Z \\
P A & P A_{1} & -P & 0 \\
(1,4)^{T} & \bar{h} Z A_{1} & 0 & -\bar{h} Z
\end{array}\right]<0} \\
{\left[\begin{array}{cc}
X & Y \\
Y^{T} & Z
\end{array}\right] \geq 0}
\end{gathered}
$$

where 


$$
\begin{aligned}
& (1,1) \triangleq-P+\bar{h} X+Y+Y^{T}+Q, \\
& (1,4) \triangleq \bar{h}(A-I)^{T} Z
\end{aligned}
$$

then the unforced system (10) with $u(k)=0$ is asymptotically stable for any time-delay $h$ satisfying $0 \leq h \leq \bar{h}$.

Proof. Choose a Lyapunov functional as follows:

$$
V(k) \triangleq V_{1}(k)+V_{2}(k)+V_{3}(k)
$$

where

$$
\begin{aligned}
V_{1}(k) \triangleq & x(k)^{T} P x(k) \\
V_{2}(k) \triangleq & \sum_{\beta=-h}^{-1} \sum_{j=k+\beta+1}^{k}[x(j)-x(j-1)] Z \\
& \times[x(j)-x(j-1)] \\
V_{3}(k) \triangleq & \sum_{j=k-h}^{k-1} x(j)^{T} Q x(j)
\end{aligned}
$$

Since it holds that

$$
x(k-h)=x(k)-\sum_{j=k-h+1}^{k}[x(j)-x(j-1)]
$$

the unforced system (10) can be written as

$$
\begin{aligned}
x(k+1)= & \left(A+A_{1}\right) x(k) \\
& -A 1 \sum_{j=k-h+1}^{k}[x(j)-x(j-1)]
\end{aligned}
$$

and thus increment of $V_{1}$ satisfies the relation

$$
\begin{aligned}
\Delta V_{1}(k)= & V_{1}(k+1)-V_{1}(k) \\
= & x(k)^{T}\left(A+A_{1}\right)^{T} P\left(A+A_{1}\right) x(k) \\
& -2 x(k)^{T}\left(A+A_{1}\right)^{T} \\
& \times P A_{1} \sum_{j=k-h+1}^{k}[x(j)-x(j-1)] \\
& +[x(k)-x(k-h)]^{T} A_{1}^{T} P A_{1} \\
& \times[x(k)-x(k-h)]-x(k)^{T} P x(k)
\end{aligned}
$$

Defining $a(\cdot), b(\cdot)$, and $\mathcal{N}$ in (6) as $a(j) \triangleq x(k)$, $b(j) \triangleq x(j)-x(j-1)$ and $\mathcal{N} \triangleq\left(A+A_{1}\right)^{T} P A_{1}$ for all $j \in[k-h+1, k]$ and applying Lemma 1 will supply (12) and

$$
\begin{aligned}
\Delta V_{1}(k) \leq & x(k)^{T}\left[A^{T} P A-P+\bar{h} X+Y+Y^{T}\right] x(k) \\
& +2 x(k)^{T}\left[-Y+A^{T} P A_{1}\right] x(k-h) \\
& +x(k-h)^{T} A_{1}^{T} P A_{1} x(k-h) \\
& +\sum_{j=k-h+1}^{k}[x(j)-x(j-1)]^{T} Z[x(j)-x(j-1)]
\end{aligned}
$$

Since $\Delta V_{2}(k)$ and $\Delta V_{3}(k)$ yield the relation

$$
\begin{aligned}
& \begin{aligned}
\Delta V_{2}(k)= & h\left[(A-I) x(k)+A_{1} x(k-h)\right]^{T} Z \\
& \times\left[(A-I) x(k)+A_{1} x(k-h)\right] \\
& -\sum_{j=k-h+1}^{k}[x(j)-x(j-1)]^{T} Z[x(j)-x(j-1)]
\end{aligned} \\
& \Delta V_{3}(k)=x(k)^{T} Q x(k)-x(k-h)^{T} Q x(k-h) \\
& \text { we have }
\end{aligned}
$$

$$
\begin{aligned}
\Delta V(k) & =\Delta V_{1}(k)+\Delta V_{2}(k)+\Delta V_{3}(k) \\
& \leq\left[\begin{array}{c}
x(k) \\
x(k-h)
\end{array}\right]^{T}\left[\begin{array}{ll}
(1,1) & (1,2) \\
(1,2)^{T} & (2,2)
\end{array}\right]\left[\begin{array}{c}
x(k) \\
x(k-h)
\end{array}\right]
\end{aligned}
$$

where

$$
\begin{aligned}
(1,1) \triangleq & A^{T} P A-P+\bar{h} X+Y+Y^{T} \\
& +\bar{h}(A-I)^{T} Z(A-I)+Q \\
(1,2) \triangleq & -Y+\bar{h}(A-I)^{T} Z A_{1}+A^{T} P A_{1} \\
(2,2) \triangleq & A_{1}^{T} P A_{1}+\bar{h} A_{1}^{T} Z A_{1}-Q
\end{aligned}
$$

Then, using the Lyapunov-Krasovskii stability theorem (Hale and Lunel, 1993) and Schur complement (Boyd et al., 1994), we can conclude that the unforced system (10) is asymptotically stable if (11) and (12) hold. This completes the proof.

The proposed stability conditions (11) and (12) are linear matrix inequality (LMI) conditions. Hence, it is easy to compute the maximum upper bound of the allowable delay $\bar{h}$ using efficient convex optimization algorithms (Boyd et al., 1994).

In the following theorem, we extend Theorem 1 to design a stabilizing memoryless controller (4) for the system (10).

Theorem 2. If there exist $L, M, N, R, W$, and $V$ such that

$$
\begin{gathered}
{\left[\begin{array}{cccc}
(1,1) & -N & (3,1)^{T} & (4,1)^{T} \\
-N^{T} & -W & L A_{1}^{T} & \bar{h} L A_{1}^{T} \\
(3,1) & A_{1} L & -L & 0 \\
(4,1) & \bar{h} A_{1} L & 0 & -\bar{h} R
\end{array}\right]<0} \\
{\left[\begin{array}{cc}
M & N \\
N^{T} & L R^{-1} L
\end{array}\right] \geq 0}
\end{gathered}
$$

where

$$
\begin{aligned}
& (1,1) \triangleq-L+\bar{h} M+N+N^{T}+W, \\
& (3,1) \triangleq(A L+B V), \\
& (4,1) \triangleq \bar{h}[(A-I) L+B V],
\end{aligned}
$$

then the system (10) with the control $u(k)=V L^{-1} x(k)$ is asymptotically stable for any time-delay $h$ satisfying $0 \leq h \leq \bar{h}$.

Proof. In view of the closed-loop system of (10) with the control (4), we replace $A$ in (11) with $A+B K$. Now, pre- and postmultiply $\operatorname{diag}\left\{P^{-1}, P^{-1}, P^{-1}, Z^{-1}\right\}$ and $\operatorname{diag}\left\{P^{-1}, P^{-1}\right\}$ to (11) and (12), respectively and apply the change of variables such that $L \triangleq P^{-1}$, 
$M \triangleq P^{-1} X P^{-1}, N \triangleq P^{-1} Y P^{-1}, R \triangleq Z^{-1}, W \triangleq$ $P^{-1} Q P^{-1}$, and $V \triangleq K P^{-1}$, then we obtain (14) and (15). This completes the proof.

It is noted that the resulting conditions for synthesis problem in Theorem 2 are no more LMI conditions because of the term $L R^{-1} L$ in (15). As a result, unfortunately, we cannot find in general the global maximum $\bar{h}$ using convex optimization algorithms in this case.

An easy way to obtain a suboptimal maximal delay instead is simply setting $R=L$ in (14) and (15), which results in LMI conditions. However, if one can afford more computational efforts, better results can be obtained using an iterative algorithm presented next.

First, we define a new variable $S$ such that $L R^{-1} L \geq$ $S$ and replace the condition (15) with

$$
\left[\begin{array}{cc}
M & N \\
N^{T} & S
\end{array}\right] \geq 0, \quad L R^{-1} L \geq S .
$$

Since $L R^{-1} L \geq S$ is equivalent to $L^{-1} R L^{-1} \leq S^{-1}$, the condition (16) is equal to

$$
\left[\begin{array}{cc}
M & N \\
N^{T} & S
\end{array}\right] \geq 0, \quad\left[\begin{array}{ll}
S^{-1} & L^{-1} \\
L^{-1} & R^{-1}
\end{array}\right] \geq 0,
$$

by Schur complement (Boyd et al., 1994). Then, by introducing new variables $T, J$, and $G$, the original condition (15) can be represented as

$$
\begin{aligned}
& {\left[\begin{array}{cc}
M & N \\
N^{T} & S
\end{array}\right] \geq 0, \quad\left[\begin{array}{ll}
T & J \\
J & G
\end{array}\right] \geq 0,} \\
& T=S^{-1}, \quad J=L^{-1}, \quad G=R^{-1} .
\end{aligned}
$$

Now, using a cone complementarity problem (El Ghaoui et al., 1997), we suggest the following nonlinear minimization problem involving LMI conditions instead of the original non-convex feasibility problem of Theorem 2:

$$
\begin{aligned}
& \text { Minimizetr }(S T+L J+R G) \\
& \text { subject to }(14), \\
& {\left[\begin{array}{cc}
M & N \\
N^{T} & S
\end{array}\right] \geq 0, \quad\left[\begin{array}{ll}
T & J \\
J & G
\end{array}\right] \geq 0,} \\
& {\left[\begin{array}{cc}
S & I \\
I & T
\end{array}\right] \geq 0, \quad\left[\begin{array}{ll}
L & I \\
I & J
\end{array}\right] \geq 0, \quad\left[\begin{array}{cc}
R & I \\
I & G
\end{array}\right] \geq 0 .}
\end{aligned}
$$

If the solution of the above minimization problem is $3 n$, that is, $\operatorname{tr}(S T+L J+R G)=3 n$, we can say from Theorem 2 that the system (10) with the control $u(k)=V L^{-1} x(k)$ is asymptotically stable. Although it is still impossible to always find the global optimal solution, the proposed nonlinear minimization problem is easier to solve than the original non-convex feasibility problem. Actually, utilizing the linearization method (El Ghaoui et al., 1997), we can easily find a suboptimal maximal delay using an iterative algorithm presented in the following. Note that the condition (15) is used as a stopping criterion in the algorithm since it is numerically very difficult in practice to obtain the optimal solution such that $\operatorname{tr}(S T+$ $L J+R G)$ is exactly equal to $3 n$.

\section{Algorithm 1.}

(1) Choose a sufficiently small initial integer $\bar{h}>0$ such that there exists a feasible solution to the LMI conditions in (17). Set $\bar{h}_{s o}=\bar{h}$.

(2) Find a feasible set $\left(J_{0}, G_{0}, L_{0}, M_{0}, N_{0}, R_{0}, S_{0}, T_{0}, V_{0}, W_{0}\right)$ satisfying (14) and (17). Set $k=0$.

(3) Solve the following LMI problem for the variables $(J, G, L, M, N, R, S, T, V, W)$ :

$$
\begin{gathered}
\text { Minimize } \operatorname{tr}\left(S_{k} T+T_{k} S+L_{k} J+J_{k} L\right. \\
\left.+R_{k} G+G_{k} R\right) \\
\text { subject to (14) and (17). }
\end{gathered}
$$

Set $J_{k+1}=J, G_{k+1}=G, L_{k+1}=L, R_{k+1}=$ $R, S_{k+1}=S$ and $T_{k+1}=T$.

(4) If the condition (15) is satisfied, then set $\bar{h}_{s o}=$ $\bar{h}$ and return to Step 2 after increasing $\bar{h}$ to some extent. If the condition (15) is not satisfied within a specified number of iterations, say $k_{\max }$, then exit. Otherwise, set $k=k+1$ and go to Step 3 .

The above algorithm gives a suboptimal maximal delay $\bar{h}_{\text {so }}$ such that the system (10) can be stabilized with the controller (4). Later, in Section 5, we shall illustrate via numerical examples that the above algorithm can provide quite satisfactory results.

\section{ROBUST STABILITY AND STABILIZATION FOR UNCERTAIN SYSTEMS}

The following theorem provides robust stability analysis of the unforced system (1) with $u(k)=0$.

Theorem 3. If there exist matrices $P>0, Q, X, Y$, $Z$ and $\Lambda=\operatorname{diag}\left\{\lambda_{1} I, \cdots, \lambda_{r} I\right\}$ such that

$$
\begin{gathered}
{\left[\begin{array}{ccccc}
P_{11} & P_{12} & A^{T} P & P_{14} & 0 \\
P_{12}^{T} & -Q+E_{1}^{T} \Lambda E_{1} & A_{1}^{T} P & \bar{h} A_{1}^{T} Z & 0 \\
P A & P A_{1} & -P & 0 & P D \\
P_{14}^{T} & \bar{h} Z A_{1} & 0 & -\bar{h} Z & \bar{h} Z D \\
0 & 0 & D^{T} P & \bar{h} D^{T} Z & -\Lambda
\end{array}\right]<0} \\
{\left[\begin{array}{cc}
X & Y \\
Y^{T} & Z
\end{array}\right] \geq 0}
\end{gathered}
$$

where

$$
\begin{aligned}
& P_{11} \triangleq-P+\bar{h} X+Y+Y^{T}+Q+E^{T} \Lambda E, \\
& P_{12} \triangleq-Y+E^{T} \Lambda E_{1}, \\
& P_{14} \triangleq \bar{h}(A-I)^{T} Z,
\end{aligned}
$$

then the unforced system (1) with $u(k)=0$ is asymptotically stable for any time-delay $h$ satisfying $0 \leq h \leq$ $\bar{h}$ and all admissible uncertainties. 
Proof. Define the left side of (11) to be $\mathcal{L}$. For uncertain system (1) with $u(k)=0$, replace $A, A_{1}$, and $B$ by $A+D \Delta E, A_{1}+D \Delta E_{1}$, and $B+D \Delta E_{b}$, respectively in condition (11). Then (11) is changed into

$$
\mathcal{L}+\bar{D} \Delta \bar{E}+\bar{E}^{T} \Delta^{T} \bar{D}^{T}<0
$$

where

$$
\begin{aligned}
& \bar{D} \triangleq\left[\begin{array}{llll}
0 & 0 & (P D)^{T} & \bar{h}(Z D)^{T}
\end{array}\right]^{T}, \\
& \bar{E} \triangleq\left[\begin{array}{llll}
E & E_{1} & 0 & 0
\end{array}\right] .
\end{aligned}
$$

From (9) in Lemma 2,

$$
\bar{D} \Delta \bar{E}+\bar{E}^{T} \Delta^{T} \bar{D}^{T} \leq \bar{D} \Lambda^{-1} \bar{D}^{T}+\bar{E}^{T} \Lambda \bar{E}
$$

and we can say that (20) holds if the following inequality is satisfied:

$$
\mathcal{L}+\bar{D} \Lambda^{-1} \bar{D}^{T}+\bar{E}^{T} \Lambda \bar{E}<0,
$$

where $\Lambda=\operatorname{diag}\left\{\lambda_{1} I, \cdots, \lambda_{r} I\right\}>0$. After some manipulation using Shcur complement, inequality condition (22) is changed to (18). This completes the proof.

Next, we extend Theorem 3 to design a robust stabilizing memoryless controller (4) for the system (1) in the following theorem.

Theorem 4. If there exist matrices $L, M, N, R, V$, $W$ and $\Lambda=\operatorname{diag}\left\{\lambda_{1} I, \cdots, \lambda_{r} I\right\}$ such that

$$
\begin{gathered}
{\left[\begin{array}{ccccc}
Q_{11} & -N & Q_{13} & Q_{14} & Q_{15} \\
-N^{T} & -W & L A_{1}^{T} & \bar{h} L A_{1}^{T} & L E_{1}^{T} \\
Q_{13}^{T} & A_{1} L & Q_{33} & \bar{h} D \Lambda D^{T} & 0 \\
Q_{14}^{T} & \bar{h} A_{1} L & \bar{h} D \Lambda D^{T} & Q_{44} & 0 \\
Q_{15}^{T} & E_{1} L & 0 & 0 & -\Lambda
\end{array}\right]<0} \\
{\left[\begin{array}{cc}
M & N \\
N^{T} & L R^{-1} L
\end{array}\right] \geq 0}
\end{gathered}
$$

where

$$
\begin{aligned}
& Q_{11} \triangleq-L+\bar{h} M+N+N^{T}+W, \\
& Q_{13} \triangleq(A L+B V), \\
& Q_{14} \triangleq \bar{h}[(A-I) L+B V]^{T}, \\
& Q_{15} \triangleq\left(E L+E_{b} V\right)^{T}, \\
& Q_{33} \triangleq-L+D \Lambda D^{T}, \\
& Q_{44} \triangleq-\bar{h} R+\bar{h}^{2} D \Lambda D^{T},
\end{aligned}
$$

then the system (1) with the control $u(k)=V L^{-1} x(k)$ is asymptotically stable for any time-delay $h$ satisfying $0 \leq h \leq \bar{h}$ and all admissible uncertainties.

Proof. Define the left side of (14) to be $\mathcal{M}$. For uncertain system (1), replace $A, A_{1}$, and $B$ by $A+$ $D \Delta E, A_{1}+D \Delta E_{1}$, and $B+D \Delta E_{b}$, respectively in condition (14). Then (14) is changed into

$$
\mathcal{M}+\bar{D} \Delta \bar{E}+\bar{E}^{T} \Delta \bar{D}<0,
$$

where

$$
\begin{aligned}
& \bar{D} \triangleq\left[\begin{array}{llll}
0 & 0 & D^{T} & \bar{h} D^{T}
\end{array}\right]^{T}, \\
& \bar{E} \triangleq\left[\begin{array}{llll}
\left(E L+E_{b} V\right) & E_{1} L & 0 & 0
\end{array}\right] .
\end{aligned}
$$

From (8) in Lemma 2,

$$
\bar{D} \Delta \bar{E}+\bar{E}^{T} \Delta \bar{D} \leq \bar{D} \Lambda \bar{D}^{T}+\bar{E}^{T} \Lambda^{-1} \bar{E}
$$

and we can say that (14) holds if the following inequality is satisfied:

$$
\mathcal{M}+\bar{D} \Lambda \bar{D}^{T}+\bar{E}^{T} \Lambda^{-1} \bar{E}<0,
$$

where $\Lambda=\operatorname{diag}\left\{\lambda_{1} I, \cdots, \lambda_{r} I\right\}>0$. After some manipulation using Shcur complement, inequality condition (26) is changed to (23). This completes the proof.

\section{NUMERICAL EXAMPLES}

First, we present a numerical example illustrating the proposed stability criteria given in Theorem 1. Let us consider a nominal state-delayed system (10) with system matrices

$$
A=\left[\begin{array}{cc}
0.8 & 0 \\
0 & 0.91
\end{array}\right], \quad A_{1}=\left[\begin{array}{cc}
-0.1 & 0 \\
-0.1 & -0.1
\end{array}\right]
$$

Any delay-independent stability criterion fails to verify that the system is asymptotically stable. Applying Theorem 1, we obtain $\bar{h}=41$.

The next example deals with the robust stabilization using Theorem 4. Let us consider a uncertain statedelayed system (10) with system matrices

$$
\begin{gathered}
A=\left[\begin{array}{cc}
1 & 0 \\
0 & 1.01
\end{array}\right], A_{1}=\left[\begin{array}{cc}
-0.02 & 0.005 \\
0 & -0.01
\end{array}\right], B=\left[\begin{array}{c}
0 \\
0.01
\end{array}\right], \\
D=0.2 \times I \quad E=E_{1}=I, \quad E_{b}=0,
\end{gathered}
$$

Using Algorithm 1, we can obtain results as shown in Table 1.

Table 1. Robust controller of Theorem 4

\begin{tabular}{|c|c|cc|}
\hline$h$ & Iterations & \multicolumn{2}{|c|}{ Feedback gain } \\
\hline \hline 10 & 2 & {$\left[\begin{array}{ll}-0.0573 & -5.3218\end{array}\right]$} \\
\hline 20 & 2 & {$\left[\begin{array}{ll}-0.1495 & -2.9303\end{array}\right]$} \\
\hline 30 & 2 & {$\left[\begin{array}{ll}-0.1442 & -2.2709\end{array}\right]$} \\
\hline 40 & 8 & {$\left[\begin{array}{ll}-0.1697 & -1.9990\end{array}\right]$} \\
\hline 41 & 40 & {$\left[\begin{array}{ll}-0.6311 & -2.3615\end{array}\right]$} \\
\hline
\end{tabular}

Note that the number of iterations in the table denotes after how many iterations the stopping criterion, i.e. the condition (15), was activated. Figure 1 compares the state trajectories of the open-loop unforced systems and the closed-loop systems controlled by the proposed controller. It clearly shows that the system is stabilized by the proposed controller.

\section{CONCLUSIONS}

This paper proposed a new robust stabilization method for a class of discrete-time systems with normbounded uncertainties and unknown constant statedelay. An algorithm involving convex optimization 


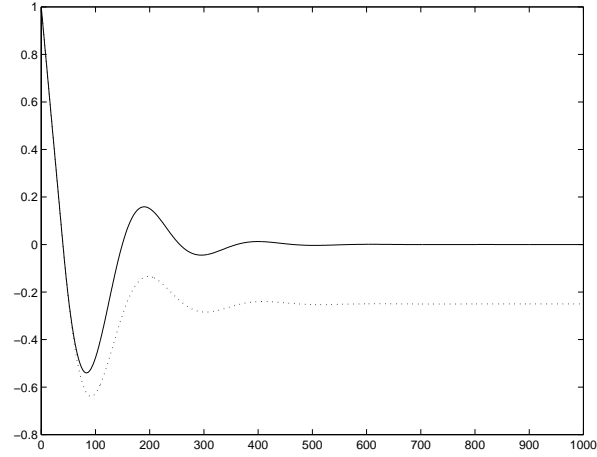

Fig. 1. State trajectories $x_{1}$ (solid line : closed-loop, dotted line : open-loop )

was also proposed to construct a controller with a suboptimal upper bound of the delay such that the system can be stabilized for all admissible uncertainties. It was shown by numerical examples that the proposed delay-dependent stabilization method can even capture the delay-independent stabilizability of the system. It is considered that the proposed results will be helpful in the analysis and synthesis for networked control systems, which inevitably include a time-delay induced by data transmission.

\section{REFERENCES}

Boyd, S., L.E. Ghaoui, E. Feron and V. Balakrishnan (1994). Linear Matrix Inequalities in System and Control Theory. Vol. 15. SIAM. Philadelphia, PA.

El Ghaoui, L., F. Oustry and M. Ait Rami (1997). A cone complementarity linearization algorithms for static output-feedback and related problems. IEEE Trans. Automat. Contr. 42(8), $1171-1176$.

Fu, M., H. Li and S. I. Niculescu (1998). Robust stability and stabilization of time-delay systems via integral quadratic constraint approach. In: Stability and Control of Time-delay Systems (L. Dugard and E. Verriest, Eds.). Springer-Verlag, London. pp. $101-116$.

Hale, J. K. and S. M. V. Lunel (1993). Introduction to Functional Differential Equations. SpringerVerlag. New York.

Kapila, V. and W. M. Haddad (1998). Memoryless $H_{\infty}$ controllers for discrete-time systems with time delay. Automatica 34(9), 1141-1144.

Kim, J. H., E. T. Jeung and H. B. Park (1996). Robust control for parameter uncertain delay systems in state and control input. Automatica 32(9), 1337 -1339 .

Lee, J. H., S. W. Kim and W. H. Kwon (1994). Memoryless $H_{\infty}$ controllers for state delayed systems. IEEE Trans. on Autom. Contr. 39, 159 - 162.

Li, X. and C. E. de Souza (1997a). Criteria for robust stability and stabilization of uncertain linear systems with state delays. Automatica 33(9), 1657 1662.

Li, X. and C. E. de Souza (1997b). Delay-dependent robust stability and stabilization of uncertain linear delay systems: a linear matrix inequality approach. IEEE Trans. Automat. Contr. 42(8), 1144 - 1148.

Li, X., C. E. de Souza and A. Trofino (1998). Delaydependent robust stabilization of uncertain linear state-delayed systems via static output feedback. In: IFAC International Workshop on Linear Time Delay Systems. Grenoble, France. pp. $1-6$.

Mahmoud, M. S. (2000). Robust $H_{\infty}$ control of discrete systems with uncertain parameters and unknown delays. Automatica 36, 627-635.

Mahmoud, M. S. and N. F. Al-Muthairi (1994). Quadratic stabilization of continuous time systems with state-delay and norm-bounded timevarying uncertainties. IEEE Trans. on Autom. Contr. 39(10), 2135 - 2139.

Niculescu, S., C. E. de Souza, J. Dion and L. Dugard (1994). Robust stability and stabilization of uncertain linear systems with state delay: Single delay case. In: IFAC Symp. Robust Control Design. Rio de Janeiro, Brazil.

Niculescu, S., E. Verriest, J. Dion and L. Dugard (1998). Stability and robust stability of timedelay systems: A guided tour. In: Stability and Control of Time-delay Systems (L. Dugard and E. Verriest, Eds.). Springer-Verlag, London. pp. 1 -71 .

Phoojaruenchanachai, S. and K. Furuta (1992). Memoryless stabilization of uncertain time-varying state delays. IEEE Trans. on Autom. Contr. 37(7), $1022-1026$.

Song, S. H., J. K. Kim, C. H. Yim and H. C. Kim (1999). $H_{\infty}$ controll of discrete-time linear systems with time-varying delays in state. Automatica 35, 1587-1591.

Xie, L. and C. E. de Souza (1993). Robust stabilization and disturbance attenuation for uncertain delay systems. In: Proc. European Contr. Conf.. Gröningen, Netherland. 